\title{
The effect of mass loading on spurious modes in micro-resonators
}

\author{
Jérôme Charmet ${ }^{1}$, Ronan Daly ${ }^{2}$, Pradyumna Thiruvenkatanathan ${ }^{1}$, and Ashwin A. Seshia ${ }^{1}$ \\ ${ }^{1}$ Nanoscience Centre, Engineering Department, University of Cambridge, \\ 11 J.J. Thomson Avenue, Cambridge, CB3 OFF, UK and \\ ${ }^{2}$ Institute for Manufacturing, Engineering Department, \\ University of Cambridge, Charles Babbage Road, Cambridge, CB3 OFS, UK
}

(Dated: July 13, 2015)

\begin{abstract}
Dissipation mechanisms severely compromise the performance of micro-resonator based sensors. In this letter we specifically examine the shift in resonant frequency of spurious modes towards the mode of interest during mass loading. This can result in modal interaction that degrades the response of the sensor. However, by understanding and controlling this effect we can overcome this key barrier to micro-resonator applications.
\end{abstract}

PACS numbers: 85.85.+j, 46.40.Ff, 07.07.Df

The prospect of mass-produced, low cost arrays of sensors integrated with modern semiconductor electronics has driven the development of a variety of micro-electromechanical-system (MEMS) sensors. Among these, micro/nano-resonators have been extensively researched as gravimetric sensors for biosensing [1-8], environmental and industrial monitoring $[9,10]$, studying the evaporation of droplets $[11,12]$ and adherent cell growth [13] as well as the evaluation of the visco-elastic properties of complex liquids such as blood $[14,15]$. In order to design optimal sensors, it is important to identify and minimize the energy dissipation mechanisms that can degrade their performance.

Losses in mechanical resonators can arise from a number of sources, including viscous drag, squeeze film damping, acoustic radiation loss via substrate attachments, mode coupling with spurious modes and other internal loss mechanisms (see for example [5] and [16]). Laterally excited bulk acoustic resonators (LBARs) actuated electrostatically have relatively low losses compared to other micro-resonators. Their patterned electrodes and design promote the selective excitation of in-plane modes, reducing the viscous drag. This configuration also minimises the risk of exciting spurious modes. However, even though their occurrence is rare, the appearance of spurious modes in LBAR is unpredictable and can result in modal interaction that severely degrades the response of the resonator [18].

In the present letter we show that substantial mass loading can shift spurious modes towards the mode of interest even if the former was not present in the unloaded condition. This proximity can result in modal interaction that severely degrades the performance of the resonator. LBARs were subjected to localised liquid loading in the form of inkjet-deposited drops to explore this phenomenon further. The results and interpretation of the phenomenon can be extended to any micro/nanomechanical resonators subjected to mass loading in the presence of spurious modes. Our experimental evidence is also supplemented by finite element modeling (FEM).
It was previously reported that FEM cannot accurately model higher order out-of-plane mode in thin resonators (see [18] for the case of LBAR), but it does provide a qualitative insight into the effect of mass loading on spurious modes.

A two port measurement set-up was used to conduct open-loop electrical characterisation of square resonators (825 $\mu \mathrm{m}$ side length and $25 \mu \mathrm{m}$ thickness) using an Agilent $4936 B$ network analyzer. The resonators, fabricated by MEMSCAP in a SOI MEMS process, were electrostatically excited in the square extension mode using a variable bias voltage $V_{d c}$ and a frequency-swept harmonic AC excitation power of $0 \mathrm{dBm}$. The measurements were conducted in a Lakeshore probe station, under vacuum $\left(\sim 1 \times 10^{-3}\right.$ mbar $)$ at $300 \mathrm{~K}$. For the purpose of the studies, a Dimatix Materials Printer DMP2800 was used to deposit liquid drops on the resonators. A solution of polyethylene glycol 200 (PEG200), was chosen as its low vapour pressure permits characterisation of the resonators under vacuum for maximum resolution. The finite element modeling (FEM) simulations were performed using COMSOL with the Solid Mechanics module (eigenfrequency study) and the "added mass" boundary condition. The added mass is defined as $m_{d}=\rho V$ where $V$ is the volume of the drop and $\rho$ the density of the liquid. The radius of the drop on the resonator is given by: $r=(3 V / 2 \pi)^{1 / 3}$ in the case of a hemisphere, which is a reasonable approximation in our case as verified visually.

PEG200 drops of 17 picolitre (pl) were deposited at the centre of the resonator, which was then excited in square extension mode (see Figure 1). The resonance frequency of the square extension mode does not change significantly for volumes of up to $500 \mathrm{pl}$ because the drop is at a nodal area. However, for volumes around $400 \mathrm{pl}$, a spurious mode appears in the vicinity of the square extension mode. Figure 2, that represents the amplitude response of the resonator, shows the case of a 425 pl drop of PEG200 deposited at the centre of the resonator. The observed time dependent positive frequency shift of the spurious mode (indicated by the arrows) is 

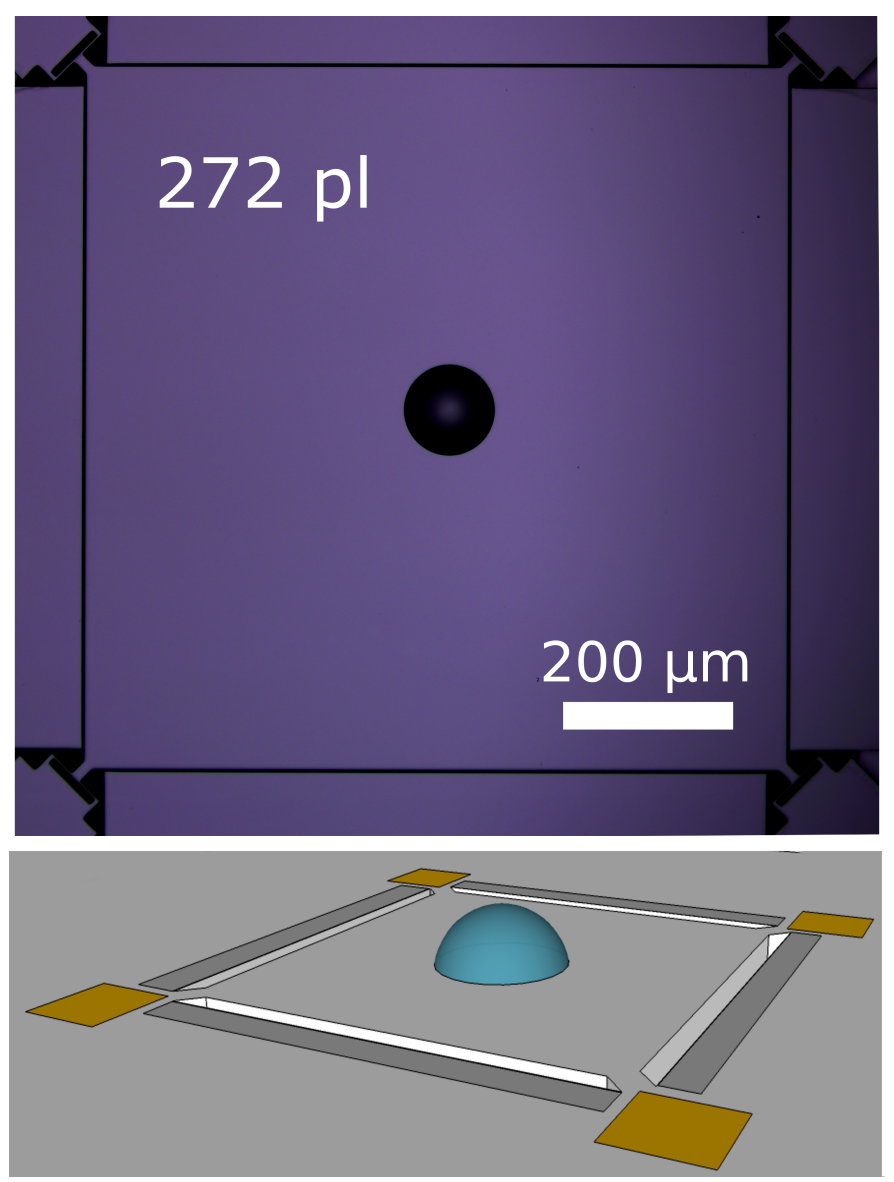

FIG. 1: Drop of PEG200 at the centre of a square resonator. Top) image showing a $272 \mathrm{pl}$ drop deposited by inkjet printing (top view). Bottom) sketch of a drop on a resonator.

due to the slow degassing of PEG200 at low pressure. Even though polyethylene glycol 200 has a low vapour pressure it is also hygroscopic (i.e. it attracts and holds water molecules from ambient air) and it may contain volatile oligomers that evaporate under vacuum. Therefore, the volume of the drop decreases under vacuum as confirmed by observation of the drops before and after the measurement.

The volume change noted reduces the effective mass of the drop and results in a positive frequency shift of the out-of-plane spurious mode. This is due to the fact that the drop is not at a nodal area of the out-of-plane spurious mode. Indeed, at the fundamental frequency of the square extension mode, around $5 \mathrm{MHz}$ in this case, the out-of-plane spurious modes are of a higher order and therefore their mode shape is expected to be quite complex with nodes and anti-nodes covering most of the surface of the resonator. One consequence of this is that a change in mass anywhere on the surface will be reflected by a frequency shift of the higher order spurious mode. In particular, a change in the surface area of the drop affects the frequency response due to the spatial variation of the

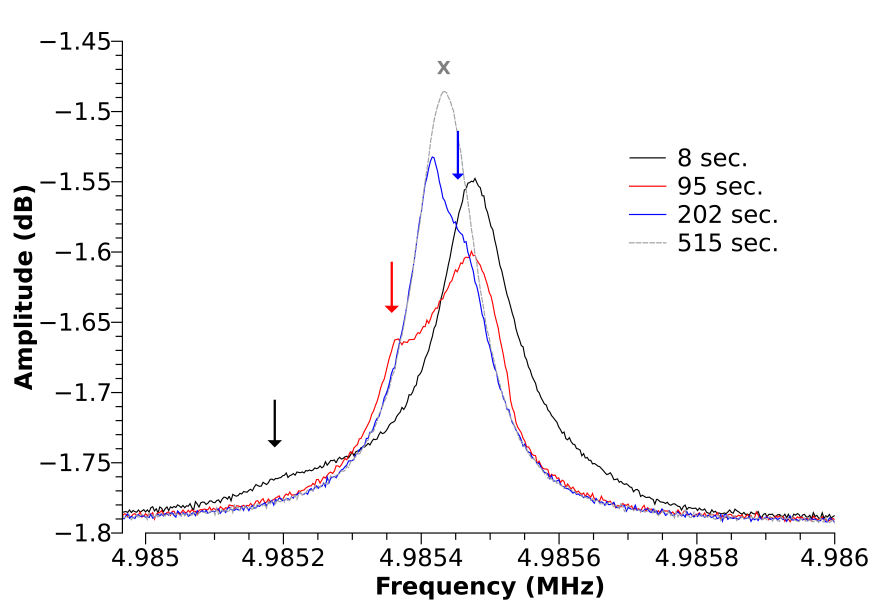

FIG. 2: Spurious mode in the vicinity of the square extension mode due to a $425 \mathrm{pl}$ drop of PEG200 at the centre of the resonator. The time dependent frequency shift of the spurious mode (indicated by the arrows) is due to the degassing of the PEG200 drop. After 515 seconds the volume loss of the drop is such that the spurious mode is not visible any more, revealing the unaffected square extension mode (indicated by $\mathrm{X}$ ). The graph shows the amplitude response (after capacitive feed-through cancellation) of a $25 \mu \mathrm{m}$ thick resonator excited in square extension mode at $\mathrm{V}_{d c}=60 \mathrm{~V}$.

mode shape. The effect of the weight and position of the drop will be examined again later using FEM simulations.

In Figure 2, the amplitude response of the unaffected square extension mode is indicated by an X. In this case, representing the measurement after 515 seconds, the decrease in volume of the drop is such that the spurious mode has shifted outside of the measurement window, leaving the square extension mode unaffected. The observed frequency shifts of the square extension mode are consistent with a curve veering phenomenon [19] and also with the FEM simulation presented below.

FEM is used in this study to provide a qualitative insight into different mass loading conditions and verify the noted observations. Figure 3 shows the resonance frequency shift of a square extension mode as a function of the drop volume. It can be seen that, for given volumes, the resonance frequency changes significantly. The frequency shifts observed are typical of a curve veering phenomenon [19] that was recently observed in MEMS [20]. This phenomenon, that results from the interaction of two weakly coupled modes, can also be observed experimentally in Figure 2. Instead of crossing, when the eigenvalues of two adjacent mode come closer, they repel one another, veer away and continue on the path that the other one would have taken had they crossed. The mode shapes in Figure 3 show clearly that an out-of-plane mode superimposes with the square extension mode for given volumes.

Figure 4 shows that the resonance frequency shift de- 


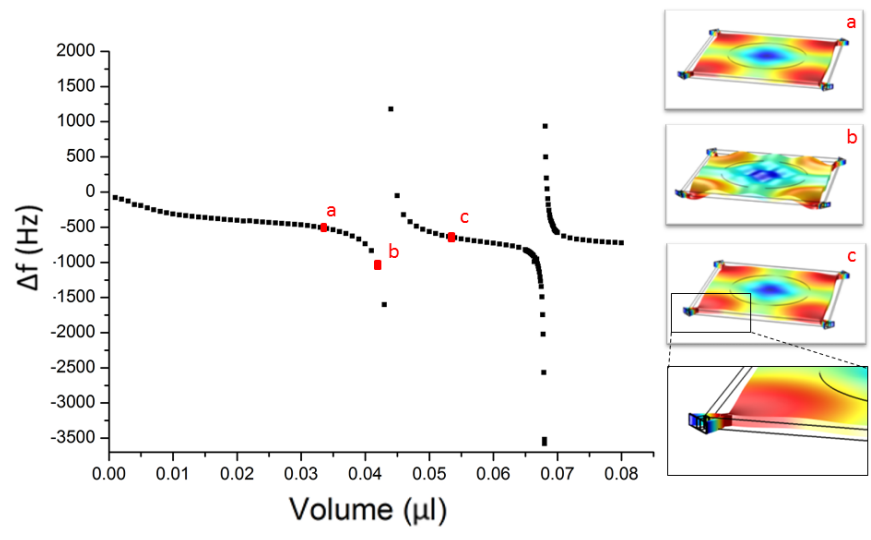

FIG. 3: FEM simulation data showing the frequency shift of the square extension mode induced by a hemispherical drop of varying volume. Each point, that corresponds to one FEM simulation, represents the Eigenfrequency of the mode of interest identified visually. The curves observed indicate a curve veering phenomenon. The two veering areas correspond to the interaction between the square extension mode and two higher order out-of-plane spurious modes. The mode shapes (right) shows the interaction with one of the spurious mode.

pends on the position as well as the weight of the drop. In the first case (Figure 4(a)), a drop of $0.1 \mu \mathrm{l}$ volume is displaced from the centre of the resonator towards one of the anchors. In the second case (Figure 4(b)), the density of a $0.1 \mu l$ drop situated at the centre of the resonator is varied from $1000 \mathrm{~kg} \mathrm{~m}^{-3}$ to $2000 \mathrm{~kg} \mathrm{~m}^{-3}$. The variations due to the manufacturing tolerances and the difficulty to position the drops exactly at the centre of the resonator combined with the results presented above account for the fact that the spurious modes were observed for slightly different volumes during each experiment. The energy exchange between the modes observed experimentally (as shown in Figure 2) corroborates the hypothesis of coupling between the modes (as mentioned above, curve veering is a feature of weakly coupled systems). However, as mentioned in [18], it is not possible to selectively excite and interrogate out-of-plane modes using the conventional LBAR transduction schemes; therefore, the precise nature of the interaction between the modes cannot be confirmed experimentally.

The observations described in this manuscript show that substantial mass perturbation, in our case induced by picolitre-scale drop deposition, can shift the resonant frequency of a spurious mode towards the mode of interest. This proximity can result in modal interaction which significantly degrades the performance of a resonator [18]. Therefore, it is important to understand the different factors that can induce modal interaction. The effect of mass loading is particularly important for sensing applications where micro-resonators are expected to be subjected to a range of mass loading conditions. It should also be noted that the finding in this manuscript
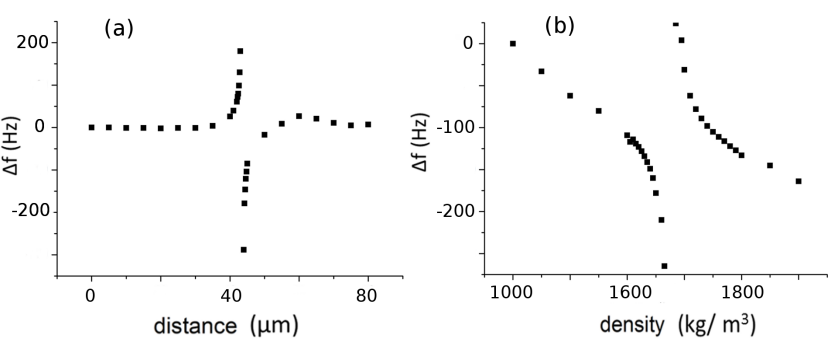

FIG. 4: FEM simulation data showing the frequency shift of the square extension mode induced by a $0.1 \mu l$ drop located at different positions from the centre of the resonator (a) and of varying density (b).

can be used to prevent modal interaction. We have shown that it is possible to selectively shift a higher order spurious mode without affecting the resonance frequency of the mode of interest by adding a mass selectively on a nodal area of the latter. This observation suggests that the same result could be achieved by depositing a thin film locally during or after the fabrication process to shift the spurious mode away from the mode of interest. Moreover, provided that the spurious mode and the mode of interest have a significantly different gravimetric sensitivity, the same result could be achieved by depositing a thin film on the whole resonator.

[1] J. Park, S. Nishida, P. Lambert, H. Kawakatsu and H. Fujita, Lab Chip, 11, 4187 (2011).

[2] E. Mehdizadeh, J. C. Chapin, J. M. Gonzales, A. Rahafrooz, R. Abdolvand, B. W. Purse, and S. Pourkamali, Sens. Actuators, A 216, 136 (2014).

[3] V. Agache, G. Blanco-Gomez, F. Baleras, and P. Caillat, Lab Chip 11, 2598 (2011).

[4] W. Xu, X. Zhang, S. Choi, and J. Chae, J. Microelectromech. Syst. 20, 213 (2011).

[5] A. T. H. Lin, J. Yan, and A. A. Seshia, J. Microelectromech. Syst. 21, 34 (2012).

[6] W. Pang, H. Zhao, E. S. Kim, H. Zhang, H. Yu, and X. Hu, Lab Chip 12, 29 (2012).

[7] F. Huber, H. P. Lang, N. Backmann, D. Rimoldi, and C. Gerber, Nat. Nanotechnol. 8, 125 (2013).

[8] J. Lee, W. Shen, K. Payer, T. P. Burg, and S. R. Manalis, Nano Lett. 10, 2537 (2010).

[9] T. Thundat, E. A. Wachter, S. L. Sharp, and R. J. Warmack, Appl. Phys. Lett. 66, 1695 (1995).

[10] J. Pettine, M. Patrascu, D.M. Karabacak, M. Vandecasteele, V. Petrescu, S.H. Brongersma, M. Crego-Calama, and C. Van Hoof, Sens. Actuators, A 189, 496 (2013).

[11] J. Arcamone, E. Dujardin, G. Rius, F. Perez-Murano, and T. Ondarcuhu, J. Phys. Chem. B. 111, 13020 (2007).

[12] A. Prasad, A. T.-H. Lin, V. R. Rao, and A. A. Seshia, Analyst, 139, 5538 (2014).

[13] K. Park, L. J. Millet, N. Kim, H. Li, X. Jin, G. Popescu, N. R. Aluru, K. Jimmy Hsia, and R. Bashir, Proc. Natl. 
Acad. Sci. U.S.A. 107, 20691 (2010).

[14] W. Xu, J. Appel, J. Chae, J. Microelectromech. Syst. 21, $302(2012)$.

[15] P. D. Smith, R. C. D. Young, and C. R. Chatwin, Measurement 43, 144 (2010).

[16] P. Mohanty, D. Harrington, K. Ekinci, Y. Yang, M. Murphy, and M. Roukes, Phys. Rev. B 66, 085416 (2002).

[17] S. Stoffels, X. Rottenberg, S. Boedecker, R. Puers, C. Rembe, and H. A. C. Tilmans, in Solid-State Sensors, Actuators and Microsystems Conference 2009, Transducers 2009, International (IEEE, 2009), pp. 14171420.

[18] J. Charmet, R. Daly, P. Thiruvenkatanathan, J. Woodhouse, and A. A. Seshia, Appl. Phys. Lett., 105, 013502 (2014).
[19] C. Pierre, J. Sound Vib. 126, 485 (1988).

[20] P. Thiruvenkatanathan, J. Woodhouse, J. Yan, and A. A. Seshia, J. Microelectromech. Syst. 20, 157 (2011).

\section{ACKNOWLEDGMENTS}

The authors would like to thank Nicola Cavaleri for proof-reading the manuscript and the Cambridge University Engineering for Clinical Practice research initiative and the W.D. Armstrong fund for financial support. 